\title{
Development of the Allocation System for Deceased Donor Liver Transplantation
}

John M. Coombes, MD and James F. Trotter, MD

As the number of pre- and post-transplant solid organ recipients continues to grow, it becomes important for all physicians to have an understanding of the process of organ procurement and allocation. In the United States, the current system for allocation and transplantation of human solid organs has been heavily influenced by the experience in deceased donor liver transplantation (DDLT). This review highlights the significant changes that have occurred over the past 10 years in DDLT, with specific attention to the impact of the Model for Endstage Liver Disease (MELD) score on organ allocation and pre- and post-transplant survival.

DDLT is managed by the United Network for Organ Sharing (UNOS) which oversees organ procurement and allocation across geographically defined Organ Procurement Organizations (OPOs). For many years, deceased donor livers were allocated to waiting list patients based on subjective parameters of disease severity and accrued waiting time. In addition, organs have traditionally been retained within the OPO where they are procured contributing to geographic disparities in disease severity at the time of transplantation among deceased donor recipients.

In response to a perceived unfairness in organ allocation, Congress issued its "Final Rule" in 1998. The Rule called for a more objective ranking of waiting list patients and more parity in disease severity among transplant recipients across OPOs. To date, little progress has been made in eliminating geographic inequities. Patients in the smallest OPOs continue to receive liver transplants at a lower level of disease severity. However, strides have been made to standardize assessments of disease severity and better prioritize waiting list patients. The MELD score has emerged as an excellent predictor of short-term mortality in patients with advanced liver disease, and patients listed for liver transplantation are now ranked based on their respective MELD scores. This has improved organ access to the most severely ill patients without compromising waiting list mortality or post-transplant survival.

The current system for DDLT remains imperfect but has improved significantly in the past decade. As the number of patients in need of DDLT grows, the system will continue to evolve to meet this increasing demand.

Keywords: Liver transplantation; Cadaver; Acute liver failure; Decision support techniques;

Tissue and organ procurement/organization \& administration; Organ transplantation; Registries.

Reprint Requests: John M. Coombes, MD, Division of Gastroenterology/Hepatology, University of Colorado Health Sciences Center, 4200 East $9^{\text {th }}$ Avenue, B- 154, Denver, CO 80262. Tel: 303-372-8866. Fax: 303-372-8868. Email: John.Coombes@UCHSC.edu.
Received: September 7, 2004

Resubmitted: December 17, 2004

Revised: March 14, 2005

Accepted: March 25, 2005 
B United States

Although it has been more than 40 years since the first human liver transplantation, ${ }^{1}$ regulation of organ allocation has developed and evolved over the last 2 decades. The first national regulation occurred in 1984 when Congress passed the National Organ Transplant Act, which created the Organ Procurement and Transplantation Network (OPTN). This network monitors all patients listed for organ transplantation and is responsible for overseeing the procurement, allocation, and transplantation of solid organs across the country. The United Network for Organ Sharing (UNOS) is the more commonly recognized contractor that manages OPTN duties on a daily basis. ${ }^{2}$

Patients requiring a liver transplant are referred to a liver transplant center, of which there are approximately 140 in the United States. After extensive evaluation, patients accepted for transplantation at each individual center are listed on the UNOS waiting list. Each center is, in turn, a member of a local Organ Procurement Organization (OPO) that is responsible for the retrieval, preservation, and transportation of donor livers to the appropriate regional transplant centers. There are 62 OPOs operating in the United States. Each OPO is comprised of between 1 and 8 liver transplantation centers. When a donor organ becomes available within an OPO, the organ must be matched to a recipient with the highest need. The current national allocation policy dictates that, in general, a deceased donor liver is allocated to the patient with the highest model for end-stage liver disease (MELD) score within the OPO where the organ was procured. The most notable exception is acute liver failure where the organ is usually allocated to the sickest patient in the UNOS region (comprised of several contiguous states). However, acute liver failure is an uncommon indication for transplantation. The prioritization of patients on the transplant list has undergone a major paradigm shift over the past decade. This evolution and the reasons behind the changes are discussed below.

Deceased-Donor Liver Allocation in the Pre-MELD Era As with all solid organs, the demand for deceased donor liver transplantation (DDLT) vastly exceeds the supply. Currently, there are over 17,000 patients listed for liver transplantation in the UNOS database, yet only 5,600 deceased donor grafts became available in 2003. This disparity between supply and demand has widened every year since 1994, thus necessitating a system to prioritize the vast number of patients waiting on a limited pool of donor organs. Over the years there have been several fundamental changes in the prioritization of patients for liver transplantation.

Prior to 1997, patients waiting for DDLT in each OPO were stratified based on their hospital status and accumulated time on the waiting list. Individuals in need of liver transplantation requiring hospitalization in an intensive care unit (ICU) were given top priority, followed by hospitalized non-ICU patients and, finally, ambulatory outpatients. With so many patients being assigned to one of only three categories, clearly there needed to be another measure of each individual's urgency for transplantation. That measure, for many years, was accrued waiting list time. Thus, although organs were fairly allocated first to sicker ICU patients over non-ICU and ambulatory patients, within each tier waiting list time was of utmost importance. To improve this advantage, many patients with chronic liver disease were listed early, while still well-compensated, simply to start accumulating time on the waiting list. ${ }^{3}$

UNOS further modified its listing criteria in 1998. The updated criteria relied heavily on the Child-Turcotte-Pugh (CTP) score that combines a number of clinical and biochemical parameters into an overall score of $\mathrm{A}, \mathrm{B}$, or $\mathrm{C}$ with progression of disease severity from A to C (table 1). By incorporating the CTP score and estimated life expectancy as predictors of disease severity, the new UNOS scoring system hoped to better stratify patients on the liver transplant list. UNOS status 1 patients were those who, in general, had acute fulminant hepatic failure and imminent risk of death without urgent liver transplantation. These patients received top priority for available livers over other patients with chronic liver disease, who were categorized as status 2A, 2B, or 3 based on their hospital status, CTP score, and presence of sequelae of end stage liver disease ${ }^{3}$ (figure 1).

Although an improvement, the new UNOS status system remained imperfect. Many factors used to calculate status (e.g., life expectancy, CTP score severity of ascites and encephalopathy) required subjective clinical assessments. Thus, although ostensibly there was a progression of disease severity from status 3 to $2 \mathrm{~B}$ to $2 \mathrm{~A}$, no guarantee existed that patients in each higher category were truly in more urgent need of liver transplantation. While fulminant status 1 patients appropriately had first chance at available livers, the much larger pool of chronic liver patients remained stratified by individual physician assessments that could not be standardized.

Table 1. Child-Turcotte-Pugh calculation.

\begin{tabular}{lccc}
\hline & \multicolumn{3}{c}{ Scoring Points } \\
\cline { 2 - 4 } & $\mathbf{1}$ & $\mathbf{2}$ & $\mathbf{3}$ \\
\hline INR & $<1.7$ & $1.7-2.3$ & $>2.3$ \\
Bilirubin, mg/dl & $<2.0$ & $2.0-3.0$ & $>3.0$ \\
Albumin, g/dl & $>3.5$ & $2.8-3.5$ & $<2.8$ \\
Encephalopathy grade & None & $1-2$ & $3-4$ \\
Ascites & None & Slight & Moderate \\
\hline Total Points & 5-6 points & $7-9$ points & $\geq 10$ points \\
CTP class & A & B & C \\
\hline
\end{tabular}

INR, International normalized ratio.

Adapted from: Child CG, Turcotte JG. Surgery and Portal Hypertension. In: Child CG, ed. The Liver and Portal Hypertension. Philadelphia, PA: W.B. Saunders Co.; 1964.50-64. 


\section{Organ Allocation}

\begin{tabular}{|c|c|c|}
\hline \multirow{2}{*}{$\begin{array}{l}\text { PRIORITY } \\
\text { Highest }\end{array}$} & Pre-MELD & Post-MELD \\
\hline & Status 1 & Status 1within OPO \\
\hline & & Status 1 within UNOS region \\
\hline & Status 2A & MELD $\geq 15$ within OPO \\
\hline & Status 2B & MELD $\geq 15$ within UNOS region \\
\hline & & MELD $<15$ within OPO \\
\hline$\underset{\text { Lowest }}{\downarrow}$ & Status 3 & MELD $<15$ within UNOS region \\
\hline
\end{tabular}

Figure 1. Schematic of the prioritization of deceased donor livers in the pre- and post-MELD era.

Additionally, time on the waiting list continued to prioritize patients within each status level. This perpetuated the situation where a scarce donor organ might not be allocated to the patient in greatest need. For example, all status 2A patients are not equal. An individual with ongoing hemorrhage from esophageal varices and a bilirubin of 30 might share status $2 \mathrm{~A}$ with a patient whose bilirubin is 5 but has been diagnosed with refractory ascites. If the latter patient had accumulated a longer waiting time, they could receive higher priority for an organ simply because their name was listed sooner.

Geographical disparities also existed in this modified system. Given the significant variation in OPO size and the practice of retaining an organ within the OPO where it was procured, well compensated patients in smaller OPOs often received transplants after minimal time on the waiting list. Meanwhile, a larger adjacent OPO might see poorly compensated cirrhotics die without transplantation because of the greater competition for a limited supply of organs. Further contributing to these geographic inequities were varying rates of brain death in different regions of the country, the variable efficiency of different OPOs in identifying and obtaining consent from potential donors, different referral and listing patterns among transplant centers, and varying payor-contractual obligations. ${ }^{4}$

\section{The Final Rule}

Despite the efforts of UNOS to modify its liver allocation parameters, a sense of unfairness pervaded the transplant community and the general public. How could the sickest fulminant liver failure patient with $100 \%$ 1-week mortality be denied access to a suitable organ because of an arbitrary geographic boundary? How could a more sick chronic liver failure patient lose out to a better compensated individual simply because the latter had been waiting longer? Because of these perceived inequities in liver allocations, which were extrapolated to solid organ transplantation in general, the US Department of Health and Human Services intervened in
1998 and challenged the transplant community with its "Final Rule." Essentially, this Rule sought to "assure that allocation of scarce organs [would] be based on common medical criteria, not accidents of geography."2 It hoped to level the playing field so organs would be allocated based on "patients" medical need" with "less emphasis ... placed on keeping organs in the local area where they [were] procured."2 Implicit in these objectives was the need for "standardized medical criteria ... to determine the status of a person's illness" with the ultimate goal being "to equalize waiting times among different areas of the country."2

Needless to say, the Final Rule was, by no means, the final word in solid organ transplantation. Although the principle was simple enough, no guidelines were issued on how to achieve the objectives. Moreover, many in the transplant community opposed implementation of the Rule, fearing that it would result in the closure of small transplant programs, limit access to transplantation, and decrease organ donation. ${ }^{5}$ Ultimately, in October of 1998, Congress suspended implementation of the Final Rule. At the same time the Institute of Medicine (IOM) was asked to review national organ allocation policies and determine the impact of the Rule on organ transplantation.

In 1999, the IOM announced its findings. ${ }^{2}$ Although many different facets of the Final Rule's impact were investigated, three primary recommendations surfaced that would hope to significantly restructure the liver allocation process in an effort to comply with the goals of the Rule:

1) Establishment of uniform Organ Allocation Areas (OAAs), each serving a population base of at least 9 million people.

2) Elimination of waiting time as an allocation criterion for status $2 \mathrm{~B}$ and 3 patients on the liver transplant list.

3) Establishment of an objective scoring system for prioritizing patients for liver transplantation.

The purpose of establishing large, uniform OAAs was to fulfill the Final Rule mandate of eliminating geographic inequities in the allocation process. After reviewing 68,000 records of patients on the liver transplant waiting list and performing complex statistical analyses, the IOM concluded that creating such OAAs would "substantially increase the allocation of organs to patients with more urgent need of a transplant ... without adversely affecting less sick patients."2 Essentially, the sickest patients in most dire need of liver transplantation would have similarly sized donor pools $(9$ million) to draw from. The IOM advocated that this goal be achieved through sharing agreements between existing OPOs instead of completely restructuring the system. To date, this recommendation has not been implemented.

With regards to waiting time, it had become clear that this was often an irrelevant criterion when making decisions 
about donor liver allocation. Certainly for the sickest patients (status 1 and 2A) it made sense to allocate new grafts to individuals that had been waiting the longest. However, among the larger pool of listed chronic liver patients, median waiting time was found "...not [to be] a good indicator of medical urgency or priority." 2 Instead, the IOM advocated a more objective point system based on "medical characteristics and disease prognoses" to stratify waiting list patients. ${ }^{2}$ Although the IOM left it to the transplant community to develop these more objective criteria, clearly the CTP score did not meet this standard. The hope was to find a "... continuous disease severity score system that used more objective, readily verifiable parameters, which could be validated as a measure of liver disease severity and predictor of mortality."6

\section{The Model for End-Stage Liver Disease (MELD)}

UNOS and the transplant community accepted the IOM challenge and subsequently analyzed a number of liver disease severity scoring models. The most promising appeared to be the MELD score. Originally developed as a survival statistic for cirrhotic patients undergoing transjugular intrahepatic portosystemic shunt (TIPS), the MELD incorporates 3 laboratory parameters: serum bilirubin, serum creatinine, and international normalized ratio [INR] to generate a score ranging from 6 (mild chronic liver disease) to 40 (most severe) ${ }^{7}$. MELD scores are calculated:

MELD score $=10\left(0.975 \times \log _{\mathrm{e}}\right.$ serum creatinine $[\mathrm{mg} / \mathrm{dL}]+$ $0.378 \times \log _{\mathrm{e}}$ serum bilirubin $[\mathrm{mg} / \mathrm{dL}]+1.120 \times \log _{\mathrm{e}} \mathrm{INR}$ $+0.643)$.

MELD subsequently was validated as a reliable estimate of short-term survival in chronic liver disease patients over a wide spectrum of disease etiology and severity. Moreover, the predictive accuracy of MELD was found to be essentially independent of complications related to portal hypertension of which ascites and encephalopathy are both subjective assessments. ${ }^{8,9}$ Based on these data, on February 27, 2002, UNOS abandoned the old liver allocation system in favor of the MELD. Status 1 categorization was kept, retaining top priority for these sickest fulminant liver failure patients, but the remaining hierarchy was dissolved. Instead of status $2 \mathrm{~A}$, $2 \mathrm{~B}$, and 3 , patients on the waiting list were now ranked based on their respective MELD scores, and time on the waiting list was only to be used as a tiebreaker in the unlikely event that two competing patients had identical MELD scores.

The impact of switching to the MELD score was recently investigated by Freeman et al. ${ }^{10}$ These authors analyzed liver transplantation data 1 year prior and 1 year following implementation of the MELD allocation system and discovered:
- A $12 \%$ reduction in new registrations to the liver transplant waiting list under MELD. This was attributed to elimination of waiting time as a ranking criterion as fewer patients with low MELD scores (less than 10) were being registered.

- A nonsignificant 3.5\% reduction in waiting list mortality under MELD. However, considering the changing dynamic of the waiting list population to one of higher disease severity, this reduction is likely more meaningful.

- Identical early post-transplant survival pre- and post-MELD. Although this suggests an actual improvement because, in general, sicker patients were being transplanted, the authors noted this may be a deceptive statistic when the impact of transplanting patients with hepatocellular carcinoma is considered. These patients get high priority MELD scores but have, generally, less severe liver disease at the time of transplantation.

Overall these data suggest a positive impact of the switch to the MELD score based system. However, liver allocation and transplantation remains an evolving process and several points require ongoing consideration.

\section{Areas for Improvement}

As mentioned earlier, the Final Rule sought to eliminate geographic discrepancies in organ transplantation and the IOM subsequently recommended standardized OAAs, each with a population base of 9 million individuals. The MELD score does not address this mandate. In fact, Trotter and Osgood $^{5}$ recently showed that small OPOs, those with fewer than 100 patients listed for liver transplantation, continue to transplant less severely ill patients than larger OPOs which have greater than 100 patients listed. Only 19\% of transplanted patients in the small OPOs had MELD scores $>24$ compared to $49 \%$ in large OPOs. The authors concluded that this "disparity in MELD scores ... would likely be resolved by increasing the size of the population served by the OPO." This was precisely the observation of the IOM 5 years earlier. ${ }^{5}$ Interestingly, a 2002 computer-simulated analysis suggested that geographical restructuring would have "little positive impact on overall results of liver transplantation in the United States." 4 While this issue remains unresolved, the fact remains that smaller OPOs continue to transplant patients with lower MELD scores.

The impact of transplanting patients with low MELD scores was recently highlighted by Merion et al. ${ }^{11}$ These authors noted a higher mortality during the first year post-transplant in recipients with MELD scores $<15$ at the time of transplantation as compared to candidates with MELD $<15$ that remained on the waiting list. Partly in response to these new data, UNOS has now amended the prioritization of deceased donor livers to give patients with higher MELD 
scores a better chance at transplantation (figure 1). While status 1 patients remain at highest priority, organs are subsequently offered locally within the procuring OPO and then within the UNOS region to patients with MELD scores $\geq 15$. Listed patients with MELD scores $<15$ are only eligible for deceased donor livers after these status 1 and MELD $\geq 15$ candidates have been exhausted.

Another Final Rule mandate called for standardized medical criteria to be used in prioritizing patients on the transplant waiting list. Certainly the MELD score is an improvement over the subjective CTP parameters of ascites and encephalopathy, but the new system is not perfect. The laboratory parameters (INR, creatinine, bilirubin) used to compute the MELD score are dynamic variables, and UNOS has not created a standardized normal value for each. Thus, MELD is highly dependent on the particular lab where each patient's blood work is processed. The potential impact of lab-to-lab variation was recently highlighted by Trotter et al. ${ }^{12}$ Twenty-nine patients listed for liver transplantation at a major university hospital were phlebotomized. Samples were sent to each of three clinical laboratories, including the university hospital, for analysis of INR, creatinine, and bilirubin. While the mean calculated MELD score for each patient was not significantly different between the university hospital's laboratory and one of the outside laboratories (13.6 versus 14.7), the third lab averaged 20\% higher $(17.1, P<0.03)$. This difference arose almost exclusively by variation in the INR, which averaged $26 \%$ higher in the third lab. Compared to the university hospital, re-calculation of the MELD score using data from the third laboratory resulted in an average change in priority for liver transplantation from the $58^{\text {th }}$ percentile to the $77^{\text {th }}$ percentile $(P=0.01)$. While these data represent only a small isolated sample of patients listed for liver transplantation, they illustrate the potential variability that can exist in an individual's MELD score based purely on laboratory methodologies and do not reflect changes in the patient's actual disease severity.

Serum creatinine also has the potential for wide fluctuations, even within the same laboratory, based on the patient's volume status. Patients with chronic liver disease are frequently on diuretics. Transient volume depletion can significantly elevate the serum creatinine and thus, the patient's MELD score without any progression of the underlying liver disease. UNOS has attempted to circumvent this phenomenon by requiring frequent updates in the MELD score (every 7 days for patients with MELD $\geq 25$, every 30 days for 19-24, every 90 days for 11-18, and every year for MELD $\leq 10)^{3}$ but such transient aberrations likely do not have a significant impact on organ allocation.

A final point of contention regarding the MELD relates to the prioritization of patients listed with hepatocellular carcinoma (HCC). HCC most commonly arises in the setting of chronic viral hepatitis with liver transplantation offering the best chance at cure and long-term survival. However, underlying liver function often remains well preserved in these patients. Without clinical or biochemical decompensation, these patients traditionally had low CTP scores while accumulating years on the waiting list and frequently developed growth and spread of their tumors to the point where they were no longer transplant candidates. Recognizing that liver transplantation is the treatment of choice for patients with localized, unresectable HCC, UNOS attempted to compensate for the low CTP scores of these patients by assigning them priority status $2 \mathrm{~B}$ in the pre-MELD era. ${ }^{13}$ Despite this prioritization, DDLT remained uncommon in these HCC candidates.

The conversion to MELD scores in 2002 maintained special priority for HCC patients because, like the CTP score, the MELD score of these individuals was typically quite low. Candidates with stage T1 and T2 disease were assigned MELD scores of 24 and 29, respectively. Under this new system, the HCC transplant rate increased, with more than $87 \%$ of HCC patients receiving transplant within 3 months of listing. ${ }^{13}$ This dramatic rise triggered concern of excessive prioritization for HCC candidates, and in February, 2003, UNOS decreased the priority MELD score for stage T1 and T2 patients to 20 and 24 , respectively.

Currently, HCC candidates average 0.69 years of listing prior to DDLT, a significant decrease compared to 2.28 years pre-MELD. Five-month waiting list survival has also improved in these patients from $90.3 \%$ pre-MELD to $95.7 \%$ post-MELD $(P<0.001)$, and 5-month post-DDLT survival remains unchanged. ${ }^{13}$ While there is no doubt that the MELD-based allocation system has benefited HCC candidates, questions remain about the impact that prioritizing HCC has made on patients with end stage liver disease of other etiologies. As noted earlier, overall waiting list mortality has improved in the post-MELD era, but could the priority assigned to $\mathrm{HCC}$ really be having a negative impact on non-HCC candidates? The post-MELD waiting time and pre- and post-transplant survival of patients without $\mathrm{HCC}$ remains to be investigated.

\section{Conclusion}

The process of allocation and transplantation of deceased donor livers has evolved since the first human liver transplantation in 1963. Waiting time and subjective parameters of disease severity no longer factor into the equation. The MELD score has shortened the DDLT waiting list and improved waiting list survival without adversely affecting post-transplant survival. However, geographic inequities and laboratory methodologies still compromise the system, and HCC patients may receive excessive prioritization at the expense of the remaining candidates. Nevertheless, MELD has had an overall favorable impact on DDLT. Further refinements to the system should make allocation and transplantation of deceased donor livers more equitable for the growing population of patients with end stage liver disease. 


\section{References}

1. Starzl TE, Marchioro TL, VonKaulla KN, Hermann G, Brittain RS, Waddel WR. Homotransplantation of the liver in humans. Surg Gynecol Obstet 1963;117:659-676.

2. Institute of Medicine Committee on Organ Procurement and Transplantation Policy. Organ Procurement and Transplantation: Assessing Current Policies and the Potential Impact of the DHHS Final Rule. Washington, DC: National Academy Press;1999. 1-38.

3. Everson GT. MELD: the answer or just more questions? Gastroenterology 2003;124:251-254.

4. Freeman RB, Harper AM, Edwards EB. Redrawing organ distribution boundaries: results of a computer-simulated analysis for liver transplantation. Liver Transpl 2002;8:659-666.

5. Trotter JF, Osgood MJ. MELD scores of liver transplant recipients according to size of waiting list: impact of organ allocation and patient outcomes. JAMA 2004;291:1871-1874.

6. Wiesner R, Edwards E, Freeman R, Harper A, Kim R, Kamath P, Kremers W, Lake J, Howard T, Merion RM, Wolfe RA, Krom R; United Network for Organ Sharing Liver Disease Severity Score Committee. Model for end-stage liver disease (MELD) and allocation of donor livers. Gastroenterology 2003;124:91-96.

7. Malinchoc M, Kamath PS, Gordon FD, Peine CJ, Rank J, ter Borg PC. A model to predict poor survival in patients undergoing transjugular intrahepatic portosystemic shunts. Hepatology 2000;31:864-871.

8. Kamath PS, Wiesner RH, Malinchoc M, Kremers W, Therneau TM, Kosberg CL, D'Amico G, Dickson ER, Kim WR. A model to predict survival in patients with end-stage liver disease. Hepatology 2001;33:464-470.

9. Wiesner RH, McDiarmid SV, Kamath PS, Edwards EB, Malinchoc M, Kremers WK, Krom RA, Kim WR. MELD and PELD: application of survival models to liver allocation. Liver Transpl 2001;7:567-580.

10. Freeman RB, Wiesner RH, Edwards E, Harper A, Merion R, Wolfe R; United Network for Organ Sharing Organ Procurement and Transplantation Network Liver and Transplantation Committee. Results of the first year of the new liver allocation plan. Liver Transpl 2004;10:7-15.

11. Merion RM, Schaubel DE, Dykstra DM, Freeman RB, Port FK, Wolfe RA. The survival benefit of liver transplantation. Am J Transplant 2005;5:307-313.

12. Trotter JF, Brimhall B, Arjal R, Phillips C. Specific laboratory methodologies achieve higher model for endstage liver disease (MELD) scores for patients listed for liver transplantation. Liver Transpl 2004;10:995-1000.

13. Sharma P, Balan V, Hernandez JL, Harper AM, Edwards EB, Rodriguez-Luna H, Byrne T, Vargas HE, Mulligan D, Rakela J, Wiesner RH. Liver transplantation for hepatocellular carcinoma: the MELD impact. Liver Transpl 2004;10:36-41. 